\title{
ABORDAGEM GEOMÉTRICA PARA MODULAÇÃO DE CONVERSORES TRÊS BRAÇOS NO ACIONAMENTO DE MÁQUINAS DE INDUÇÃO BIFÁSICAS SIMÉTRICAS E ASSIMÉTRICAS
}

\author{
Rodrigo Padilha Vieira* \\ rodrigovielgmail.com \\ Rodrigo Zelir Azzolin ${ }^{\dagger}$ \\ rodrigoazzolin@gmail.com
}

\author{
Cristiane Cauduro Gastaldini* \\ gastaldini@ieee.org
}

Humberto Pinheiro ${ }^{\ddagger}$

humberto. ct lab.ufsm.br@gmail.com

\author{
Hilton Abílio Gründling $\ddagger$ \\ ghilton03@gmail.com \\ ${ }^{*}$ Universidade Federal do Pampa - UNIPAMPA \\ Av. Tiarajú, 810, Alegrete, RS, Brasil. \\ ${ }^{\dagger}$ Universidade Federal do Rio Grande - FURG \\ Rio Grande, RS, Brasil. \\ ${ }^{\ddagger}$ Universidade Federal de Santa Maria - UFSM \\ Grupo de Eletrônica de Potência e Controle - GEPOC \\ Av. Roraima, 1000, Santa Maria, RS, Brasil.
}

\section{ABSTRACT}

Geometric Approach for Modulation of Three-Leg Inverters Driving Symmetrical and Asymmetrical TwoPhase Induction Machines

This paper presents a geometric approach for PWM modulation of three-leg inverters driving two-phase induction machines (TPIM). The main contribution of proposed scheme is the low computational effort to obtain symmetrical and asymmetrical line voltages suitable to fed two-phase induction machines. It is demonstrated that the amplitudes of line voltages can to vary from zero until $0.707 \mathrm{pu}$ of DClink for symmetrical output, while one of the voltages can overcome $0.707 \mathrm{pu}$ of DC-link voltage in the asymmetrical case. The results obtained with the proposed modulation technique are similar to those obtained with space vector modulation, however, the implementation of the proposed

Artigo submetido em 10/08/2010 (Id.: 01180)

Revisado em 28/12/2010, 28/04/2011

Aceito sob recomendação do Editor Associado Prof. José Antenor Pomilio modulation technique is much more simpler than the implementation of space vector modulation. Experimental results that validate the theoretical analysis are presented and they demonstrate the good performance and simplicity of the proposed modulation method.

KEYWORDS: Three-leg voltage source inverter, Two-phase induction machine, PWM modulation.

\section{RESUMO}

Este trabalho apresenta uma abordagem geométrica para modulação PWM de inversores a três braços acionando máquinas de indução bifásicas. A principal característica do esquema proposto é o número reduzido de cálculos numéricos na modulação de tensões equilibradas e desequilibradas. $\mathrm{Na}$ modulação de tensões equilibradas é demonstrado que as amplitudes de linha das mesmas podem variar de zero até 0,707 pu da tensão do barramento CC, enquanto que na modulação de tensões de linha desequilibradas a amplitude da uma 
destas pode ultrapassar o valor de 0,707 pu do barramento CC. Os resultados obtidos com a técnica proposta são semelhantes aos resultados obtidos com modulação space vector, porém, a implementação da técnica geométrica proposta neste trabalho é realizada com menor esforço computacional comparado a modulação space vector. Resultados experimentais são apresentados para validação da análise teórica, e estes confirmam o bom desempenho do método de modulação proposto.

PALAVRAS-CHAVE: Inversor de tensão de três braços, Máquina de indução bifásica, Modulação PWM.

\section{INTRODUÇÃO}

As máquinas de indução bifásicas simétricas e assimétricas (TPIM) são largamente utilizadas em aplicações de baixa potência, principalmente residenciais e comerciais. Essas máquinas normalmente são conectadas diretamente à rede de alimentação e operam em velocidade constante. Entretanto, operando dessa maneira e ainda sob certas condições de carga essas máquinas apresentam desvantagens como baixo rendimento, baixo fator de potência e pulsações de torque. Tais características têm levado muitos pesquisadores a desenvolver trabalhos relacionados a motores de melhor eficiência como: brushless DC (motores CC sem escovas) ou motor síncrono a ímã permanente. Porém, quando se considera o volume instalado e a indústria de base já consolidada, verifica-se que pesquisas relacionadas à máquinas bifásicas ainda são bem recebidas pela indústria e meio acadêmico (Blaabjerg et al., 2004; Jang, 2007).

Usualmente os equipamentos que fazem uso de máquinas de indução bifásicas são os sistemas de refrigeração, máquinas de lavar e ferramentas. Estes apresentam um consumo de energia individual que pode ser considerado baixo, no entanto o montante de todas aplicações consome uma parcela considerável da energia elétrica produzida no mundo (Wells et al., 2004). Como resultado disso várias pesquisas recentes têm apresentado sistemas de acionamento em velocidade variável aplicados a motores bifásicos. Enquanto alguns pesquisadores têm focado seus esforços no desenvolvimento de hardwares e drives para tais acionamentos (Chomat and Lipo, 2003; Donlon et al., 2002; dos Santos et al., 2010), outros pesquisadores têm desenvolvido e apresentado técnicas de controle de alto desempenho, controle vetorial e controle sensorless aplicados a máquinas bifásicas (Corrêa et al., 2000; Vaez-Zadeh and Reicy, 2005; de F. Campos et al., 2007; Vieira et al., 2009; Jemli et al., 2009; Zahedi and Vaez-Zadeh, 2009; Amin et al., 2009; Guerreiro et al., 2010).

Além do esforço realizado no desenvolvimento de leis de controle, muitos pesquisadores têm investigado técnicas para otimizar o método de modulação PWM usada no aciona- mento destas máquinas (Holmes and Kotsopoulos, 1993; Jang and Yoon, 2003; Jabbar et al., 2004; Charumit and Kinnares, 2009). Em Holmes e Kotsopoulos (1993), os autores desenvolvem uma técnica de modulação PWM de fácil implementação, que pode atuar em toda faixa da região linear do inversor modulando tensões médias que podem ter amplitude de 0,707 pu da tensão do barramento CC. Esta técnica é análoga a injeção da terceira harmônica para o caso de acionamentos de cargas trifásicas.

Por outro lado, o conceito de modulação vetorial e a sua aplicação digital são bem conhecidos em sistemas trifásicos acionando cargas trifásicas (Holmes and Lipo, 2003; Pinheiro et al., 2005). Porém, quando se considera o acionamento de cargas bifásicas, principalmente bifásicas assimétricas, o tanto a modulação e controle ainda não foram amplamente explorados (Charumit and Kinnares, 2009). Em (Jang and Yoon, 2003; Kinnares and Charumit, 2009; Jabbar et al., 2004; Martins et al., 2006) os autores desenvolvem técnicas de modulação baseadas na abordagem space vector aplicadas a conversores de dois e três braços. Entretanto, técnicas que utilizam modulação vetorial apresentam peso computacional maior, consequentemente o grande número de multiplicações e somas pode inviabilizar a implementação digital em sistemas microcontrolados de custo reduzido, e com baixa capacidade de realização de cálculos numéricos.

A abordagem geométrica para modulação de conversores PWM foi desenvolvida em (Ryan et al., 1999). No trabalho é estabelecida uma transformação linear que relaciona o espaço das tensões de saída do conversor com dimensão 2 com o espaço das tensões das fases com dimensão 3. Para definir uma relação única entre estes espaços é utilizada uma variável adicional, que pode ser sintetizada como a tensão de modo comum do conversor, ou como a tensão que representa a injeção da harmônica múltipla de três.

Neste trabalho é proposto um método geométrico aplicado na modulação de conversores trifásicos de três braços acionando cargas bifásicas simétricas e assimétricas, o qual complementa o trabalho apresentado previamente (Vieira et al., 2010). O método de modulação apresentado pode ser aplicado no acionamento de cargas equilibradas e desequilibradas. Na modulação de tensões bifásicas equilibradas as amplitudes das tensões de linha podem atingir 0,707 pu do barramento $\mathrm{CC}$, enquanto na modulação de tensões desequilibradas umas das tensões de linha pode ultrapassar 0,707 pu do barramento CC. O trabalho é organizado como segue, na Seção 2 é apresentada a descrição da técnica de modulação proposta e operação com tensões equilibradas, já a Seção 3 mostra operação com tensões assimétricas. A Seção 4 apresenta resultados experimentais obtidos com a técnica, por fim, a Seção 5 traz os principais pontos abordados no trabalho. 


\section{DESCRIÇÃO DA TÉCNICA DE MODU- LAÇÃO PROPOSTA}

A estratégia de modulação proposta neste trabalho é baseada no uso de um inversor trifásico de três braços, mostrado na Figura 1. A partir da Figura 1 pode-se definir as tensões nos pontos $a, b$, e $c$ do conversor, relacionadas com um ponto de conexão comum $g$, onde as tensões dos braços aplicadas aos interruptores $S_{4}, S_{5}$ e $S_{6}$ podem ser definidas como: $V_{a g}$, $V_{b g}$ e $V_{c g}$. As tensões de linha, ou as tensões aplicadas à carga bifásica podem ser relacionadas na forma matricial de acordo com (1).

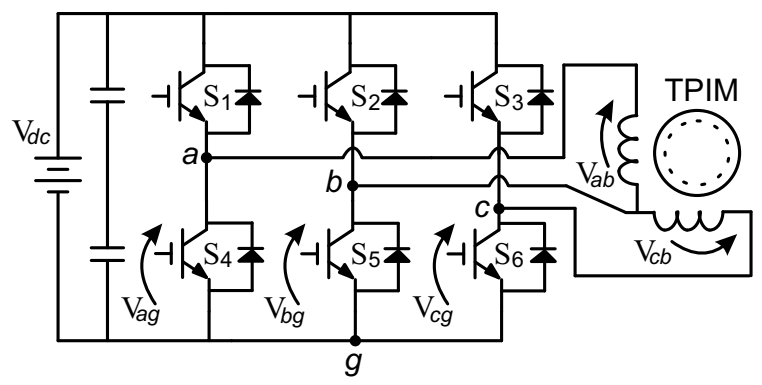

Figura 1: Inversor de três braços no acionamento de uma máquina de indução bifásica.

$$
\left[\begin{array}{l}
V_{a b} \\
V_{c b}
\end{array}\right]=\left[\begin{array}{lll}
1 & -1 & 0 \\
0 & -1 & 1
\end{array}\right]\left[\begin{array}{l}
V_{a g} \\
V_{b g} \\
V_{c g}
\end{array}\right]
$$

A equação (1) relaciona as tensões dos braços do conversor no espaço das tensões de saída. Para garantir que a matriz de transformação apresentada em (1) seja não-singular, aqui faz-se a definição de uma variável chamada de tensão de modo comum $V_{0}^{*}$, a qual é inserida como uma última linha da matriz de transformação de (1). Neste caso $V_{0}^{*}$ representa a soma das tensões nos braços do conversor, sendo mostrada em (2).

$$
V_{0}^{*}=V_{a g}+V_{b g}+V_{c g}
$$

A partir de (2) é possível reescrever (1), como,

$$
\left[\begin{array}{l}
V_{a b} \\
V_{c b} \\
V_{0}^{*}
\end{array}\right]=\left[\begin{array}{ccc}
1 & -1 & 0 \\
0 & -1 & 1 \\
1 & 1 & 1
\end{array}\right]\left[\begin{array}{l}
V_{a g} \\
V_{b g} \\
V_{c g}
\end{array}\right]
$$

Se a matriz de transformação é não-singular, (3) pode ser reescrita na forma

$$
\left[\begin{array}{l}
V_{a g} \\
V_{b g} \\
V_{c g}
\end{array}\right]=\frac{1}{3}\left[\begin{array}{ccc}
2 & -1 & 1 \\
-1 & -1 & 1 \\
-1 & 2 & 1
\end{array}\right]\left[\begin{array}{l}
V_{a b} \\
V_{c b} \\
V_{0}^{*}
\end{array}\right]
$$

Com isso é possível definir as tensões dos braços do conversor relacionadas com as tensões de linha aplicadas aos terminais da máquina e a tensão de modo comum $V_{0}^{*}$. Logo,

$$
\begin{aligned}
V_{a g} & =\frac{1}{3}\left(2 V_{a b}-V_{c b}+V_{0}^{*}\right) \\
V_{b g} & =\frac{1}{3}\left(-V_{a b}-V_{c b}+V_{0}^{*}\right) \\
V_{c g} & =\frac{1}{3}\left(-V_{a b}+2 V_{c b}+V_{0}^{*}\right)
\end{aligned}
$$

Normalizando a tensão do barramento CC do conversor de três braços em 1 pu, os limites que podem ser modulados das tensões de fase para que o inversor opere na região linear, são dados por (6).

$$
\begin{aligned}
& 0 \leq V_{a g} \leq 1 \\
& 0 \leq V_{b g} \leq 1 \\
& 0 \leq V_{c g} \leq 1
\end{aligned}
$$

A partir de (5) e (6) é possível definir os limites da tensão $V_{0}^{*}$ em função das tensões de linha desejadas $V_{a b}$ e $V_{c b}$. Logo, as seguintes desigualdades podem ser escritas,
i) $V_{0}^{*} \geq-2 V_{a b}+V_{c b}$
ii) $V_{0}^{*} \geq V_{a b}+V_{c b}$
iii) $\quad V_{0}^{*} \geq V_{a b}-2 V_{c b}$
iv) $\quad V_{0}^{*}-3 \leq-2 V_{a b}+V_{c b}$
v) $V_{0}^{*}-3 \leq V_{a b}+V_{c b}$
vi) $\quad V_{0}^{*}-3 \leq V_{a b}-2 V_{c b}$

A partir das desigualdades apresentadas em (7), com o intuito de simplificar a obtenção da tensão $V_{0}^{*}$ são definidas as seguintes funções:

$$
\begin{aligned}
r 1 & =-2 V_{a b}+V_{c b} \\
r 2 & =V_{a b}+V_{c b} \\
r 3 & =V_{a b}-2 V_{c b}
\end{aligned}
$$

Assim, para que sejam respeitadas as desigualdades estabelecidas em (7), a tensão $V_{0}^{*}$ deverá estar compreendida entre,

$$
\max \{r 1, r 2, r 3\} \leq V_{0}^{*} \leq 3+\min \{r 1, r 2, r 3\}
$$


A partir de (9) é possível verificar que existem infinitas soluções para a definição da tensão $V_{0}^{*}$, desde que a mesma satisfaça (9). Uma das soluções possíveis é igualar o valor de $V_{0}^{*}$ ao valor superior ou inferior do limite apresentado na equação. Neste caso, é possível reduzir o número de comutações. Neste trabalho o valor de $V_{0}^{*}$ é definido como a média aritmética dos valores máximos e mínimos, ou seja conforme apresentado em (10). Essa definição foi realizada com o intuído de que o valor de $V_{0}^{*}$ seja calculado de maneira simples e ainda se tenha uniformidade no número de comutações entre cada meio ciclo das tensões dos braços.

$$
V_{0}^{*}=\frac{\max \{r 1, r 2, r 3\}+(3+\min \{r 1, r 2, r 3\})}{2}
$$

A Figura 2 mostra os valores máximos e mínimos para a tensão $V_{0}^{*}$ durante a operação na região linear do conversor.

No exemplo ilustrado pela Figura 2, as amplitudes das tensões máximas de saída do conversor para uma carga equilibrada são definidas 0,68 pu do valor do barramento CC. Este valor foi escolhido para melhor visualização dos limites de $V_{0}^{*}$, porém a máxima amplitude possível das tensões de linha é 0,707 pu do barramento CC. As tensões de linha estão defasadas em $90^{\circ}$ e têm frequência de $60 \mathrm{~Hz}$.

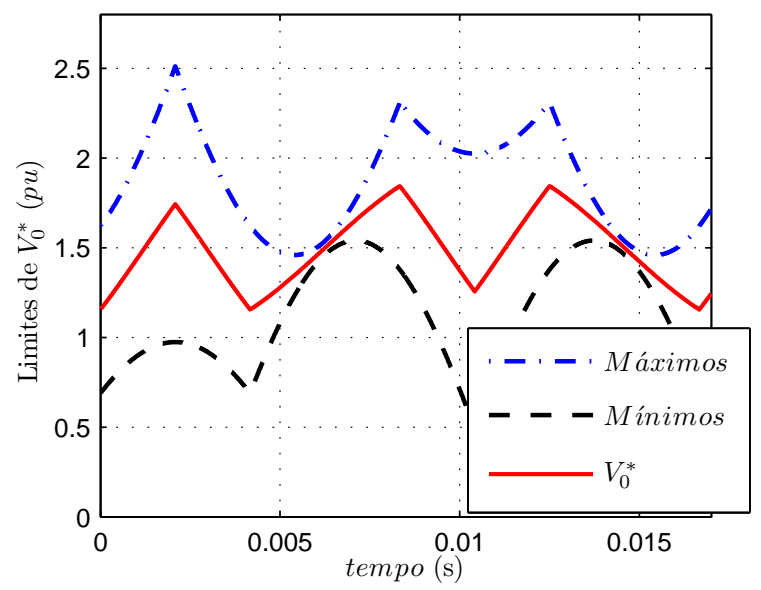

Figura 2: Valores máximos e mínimos de $V_{0}^{*}$.

As amplitudes máximas das tensões de linha moduladas pelo conversor podem ser obtidas a partir do ponto onde o valor da tensão $V_{0}^{*}$ é igual ao valor $\max \{r 1, r 2, r 3\}$, e também quando $V_{0}^{*}$ é igual a $(3+\min \{r 1, r 2, r 3\})$, como pode ser verificado na Figura 2. Neste ponto as amplitudes máximas das tensões de linha equilibradas aplicadas a carga são 0,707 pu do barramento $\mathrm{CC}$.

De forma análoga, a partir da representação do espaço das tensões de saída do conversor é possível a obtenção das amplitudes máximas das tensões de linha. Para tanto, assume-se que os estados de condução dos interruptores $S 4, S 5$ e $S 6$, bem como as tensões de linha produzidas pelo conversor são representados na Tabela 1 .

Tabela 1: Estado dos interruptores e tensões de saída correspondentes.

\begin{tabular}{cccccc}
\hline \hline$S 4$ & $S 5$ & $S 6$ & $V_{a b}$ & $V_{c b}$ & \\
\hline \hline 0 & 0 & 0 & 0 & 0 & $v_{0}$ \\
\hline 1 & 0 & 0 & 0 & 1 & $v_{1}$ \\
\hline 1 & 0 & 1 & 1 & 1 & $v_{2}$ \\
\hline 0 & 0 & 1 & 0 & 1 & $v_{3}$ \\
\hline 0 & 1 & 1 & -1 & 0 & $v_{4}$ \\
\hline 0 & 1 & 0 & -1 & -1 & $v_{5}$ \\
\hline 1 & 1 & 0 & 0 & -1 & $v_{6}$ \\
\hline 1 & 1 & 1 & 0 & 0 & $v_{7}$ \\
\hline \hline
\end{tabular}

Como consequência, é possível obter a trajetória das tensões de linha produzidas no conversor, bem como os planos limites destas tensões, mostrados na Figura 3. Com base na Figura 3 são obtidas as relações trigonométricas que mostram que as amplitudes máximas das tensões de linha devem ser 0,707 , ou $1 / \sqrt{2}$ para que o círculo que representa a trajetória das tensões fique circunscrito ao hexágono irregular que representa os planos limites do conversor mostrados na Figura 3.

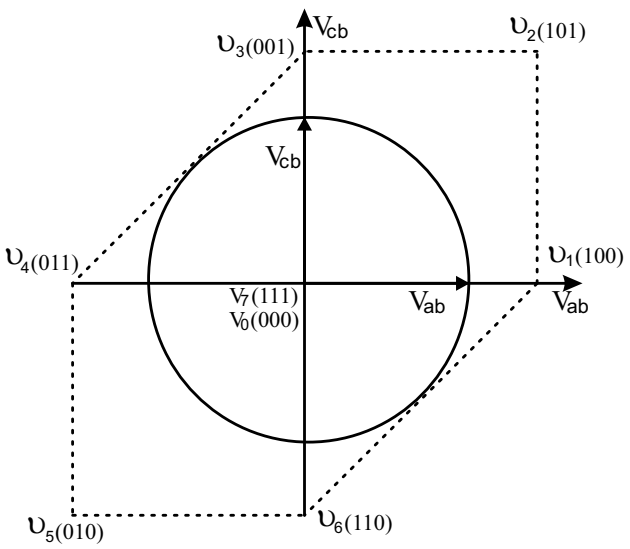

Figura 3: Espaço das tensões no plano das tensões de saída.

A Figura 4 mostra as tensões de referência $V_{a g}, V_{b g}$, e $V_{c g}$ dos braços do conversor para obtenção das tensões de linha 
equilibradas com amplitudes de saída $V_{a b}$ e $V_{c b}$ definidas em 0,68 pu do barramento $\mathrm{CC}$, e freqüências em $60 \mathrm{~Hz}$. A Figura 5 mostra as tensões de saída sintetizadas pelo conversor.

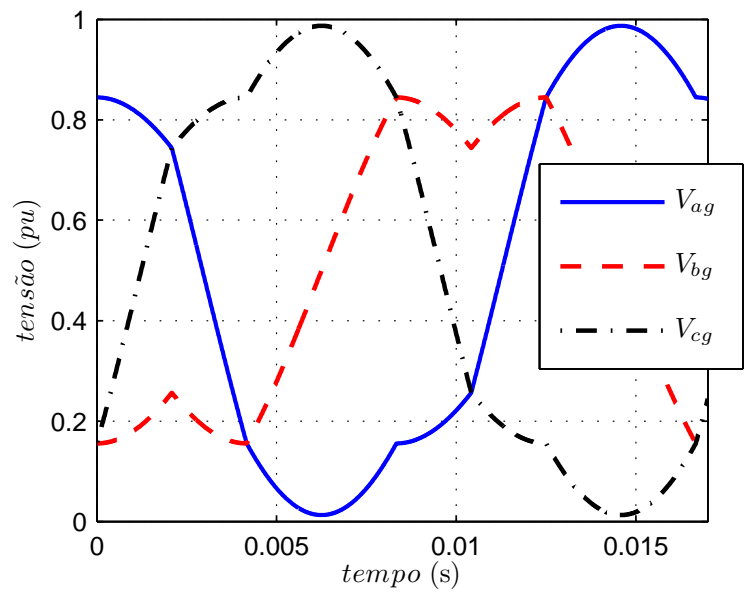

Figura 4: Tensões $V_{a g}, V_{b g}, V_{c g}$ nos braços do conversor.

Como sugerido neste trabalho, outra solução possível para determinação de $V_{0}^{*}$ é igualar o mesmo ao valor superior ou inferior do limite apresentado em (9). Neste caso, é possível reduzir o número de comutações. Para exemplificar isto, a Figura 6 mostra as tensões $V_{a g}, V_{b g}$ e $V_{c g}$ quando o valor de $V_{0}^{*}$ é definido como o mínimo de $3+(r 1, r 2$ e $r 3)$, enquanto a Figura 7 mostra as tensões nos braços do conversor para $V_{0}^{*}$ igual ao máximo de $(r 1, r 2$ e $r 3)$.

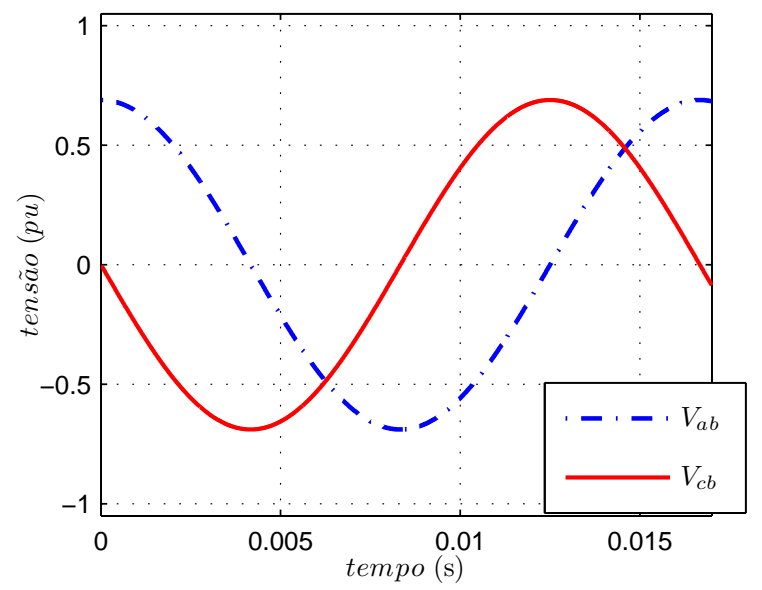

Figura 5: Tensões de linha equilibradas aplicadas a carga bifásica.

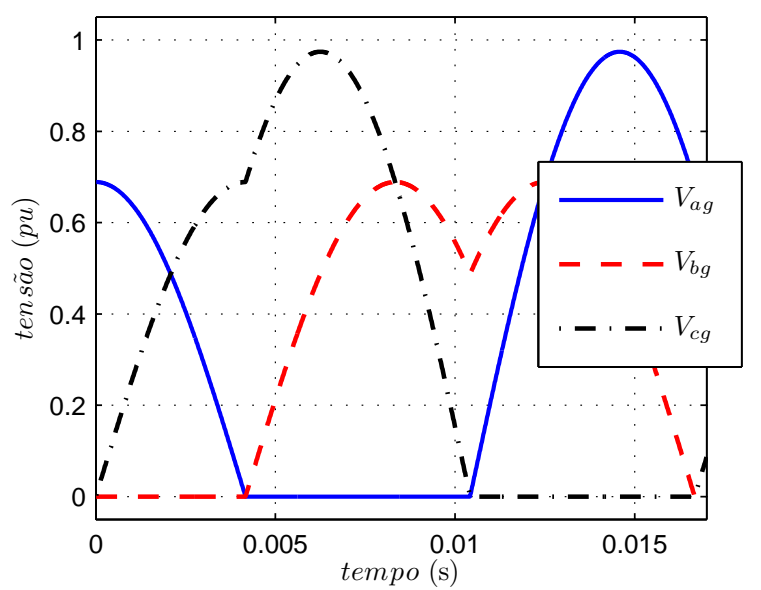

Figura 6: $V_{a g}, V_{b g}$ e $V_{c g}$ para $V_{0}^{*}$ igual ao mínimo de $3+(r 1$, $r 2$ e $r 3)$

É possível observar a partir da Figura 6 e da Figura 7 que durante os intervalos de tempo em que a tensão permanece com valor mínimo ou máximo o interruptor não deve comutar, consequentemente reduz-se o número de comutações no inversor. As tensões nos braços do conversor para estes casos produzem tensões de linha como ilustrado na Figura 5.

Com o objetivo de estabelecer um critério de comparação em função da escolha de $V_{0}^{*}$, dois parâmetros são analisados neste trabalho. O primeiro índice é o fator de distorção de primeira ordem, $D F 1$, que é um índice de desempenho proporcional à taxa de distorção harmônica das correntes de saída do inversor quando se utiliza um filtro de primeira ordem (Enjeti et al., 1990),

$$
D F 1=100 \sqrt{\sum_{n=2}^{\infty}\left(\frac{V_{n}}{n}\right)^{2}}
$$

onde $V_{n}$ é a tensão de linha de saída do conversor e $n$ é a ordem da harmônica.

A Figura 8 mostra a comparação do índice $D F 1$ para duas escolhas de $V_{0}^{*}$, uma dada por (10) e outra escolhendo $V_{0}^{*}=$ Máximos, em relação ao índice de modulação. A escolha de $V_{0}^{*}=$ Minimos tem comportamento idêntico a escolha de $V_{0}^{*}=$ Máximos. 


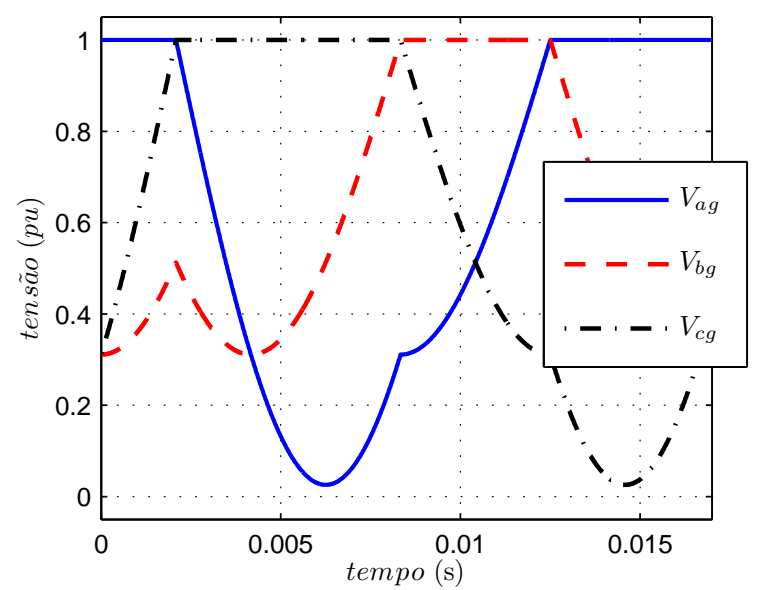

Figura 7: $V_{a g}, V_{b g}$ e $V_{c g}$ para $V_{0}^{*}$ igual ao máximo de $(r 1, r 2$ e $r 3$ )

O segundo parâmetro analisado é o número de comutações com relação ao índice de modulação, a Figura 9 mostra esta comparação considerando as comutações de $S 1$ e que a frequência de comutação do conversor seja de $5 \mathrm{KHz}$.

Com base nas equações (1) - (10) é possível verificar a realização de poucos cálculos numéricos para implementação da estratégia de modulação proposta, sendo estes, multiplicações ou somas. Se comparado a uma estratégia de modulação space vector, como a apresentada em (Kinnares and Charumit, 2009), para determinação dos tempos de comutação das chaves em cada setor do espaço das tensões são necessários vários cálculos de senos, além de multiplicações e somas. Com base na Tabela III apresentada em (Kinnares and Charumit, 2009) é possível verificar que a estratégia de modulação aqui proposta é realizada com menor esforço computacional produzindo resultados muito semelhantes.

\section{OPERAÇÃO COM TENSÕES DESEQUI- LIBRADAS}

Grande parte das máquinas bifásicas é do tipo monofásica, onde um dos enrolamentos é conectado em série com capacitor de partida ou com capacitor permanente. Nestas máquinas, os enrolamentos chamados auxiliar e principal têm impedâncias diferentes, e consequentemente, tensões desequilibradas devem ser aplicadas para reduzir as pulsações de torque. Com isso o conversor de três braços pode sintetizar uma tensão com amplitude superior a 0,707 pu do barramento CC

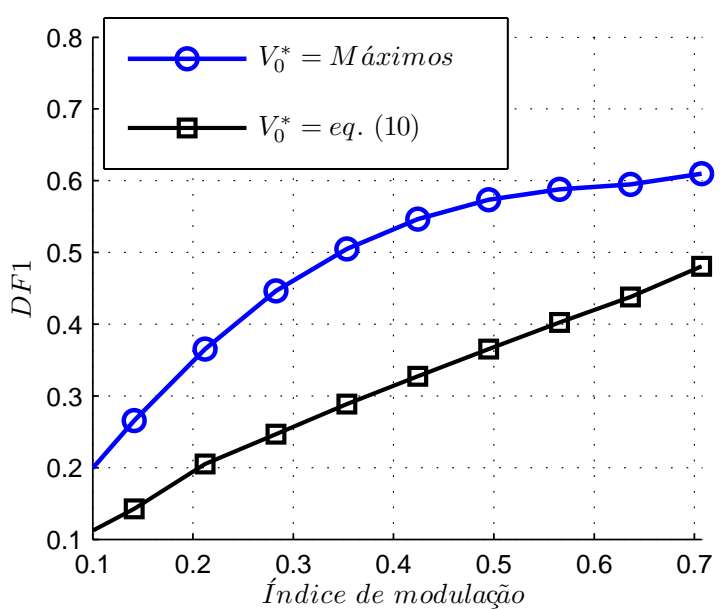

Figura 8: Índice DF1 com diferentes $V_{0}^{*}$

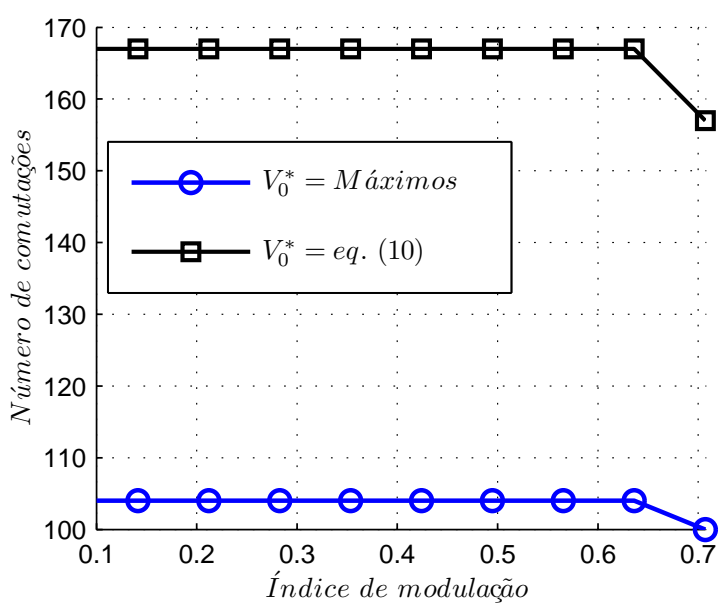

Figura 9: Número de comutações de $S 1$ para diferentes $V_{0}^{*}$

em um dos enrolamentos da máquina na região de operação linear do conversor, enquanto que no outro enrolamento uma tensão de menor amplitude é aplicada. A relação entre as amplitudes máximas sintetizadas pelo conversor pode ser obtida com base na Figura 10. Na Figura 10 é apresentado o espaço das tensões considerando que estas sejam desequilibradas. A operação na região linear do conversor requer que a elipse formada pelas tensões assimétricas de linha esteja circunscrita ao hexágono irregular que representa os planos limites das tensões do conversor, como ilustra a Figura 10, para os casos onde $V_{c b}<V_{a b}$ e $V_{c b}>V_{a b}$.

Assumindo que as tensões de linha do conversor sempre tenham defasagem de $90^{\circ}$, a partir da "Lei dos Cossenos" é 


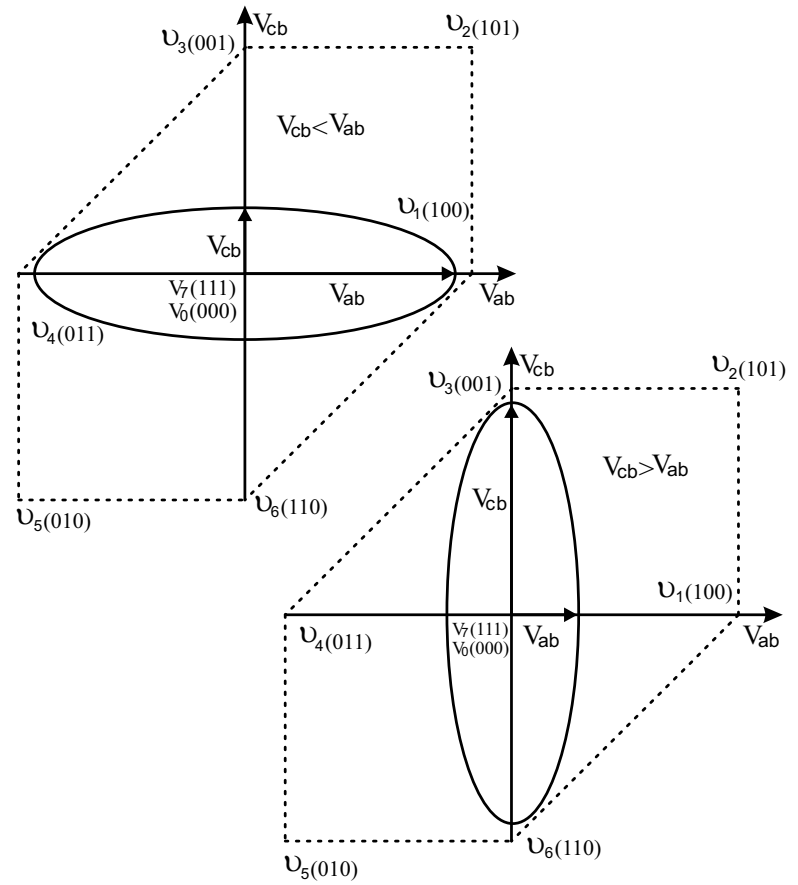

Figura 10: Espaço das tensões no plano das tensões de saída com modulação de tensões assimétricas.

possível definir a relação entre as amplitudes máximas de $V_{a b}$ e $V_{c b}$ para que a soma destes seja igual a tensão do barramento ( $1 \mathrm{pu})$, e consequentemente para que a elipse formada por estas tensões seja máxima e circunscrita ao hexágono irregular do espaço das tensões. A relação entre as amplitudes das tensões normalizadas é dada em (12).

$$
V_{a b}^{2}+V_{c b}^{2}=1
$$

A relação entre as amplitudes das tensões de saída produzidas no conversor pode ser definida como,

$$
n=\frac{V_{a b}}{V_{c b}}
$$

Considerando (12) e (13) é possível obter os valores das tensões $V_{a b}$ e $V_{c b}$ relacionadas por $n$, (14) e (15) mostram essas equações.

$$
\begin{aligned}
& V_{a b}=\sqrt{\frac{n^{2}}{n^{2}+1}} \\
& V_{c b}=\sqrt{\frac{1}{n^{2}+1}}
\end{aligned}
$$

A partir de (14) e (15) é possível traçar a curva das tensões de saída do conversor, mostrada na Figura 11, quando este opera na região linear, pela razão $n$ das tensões de saída.

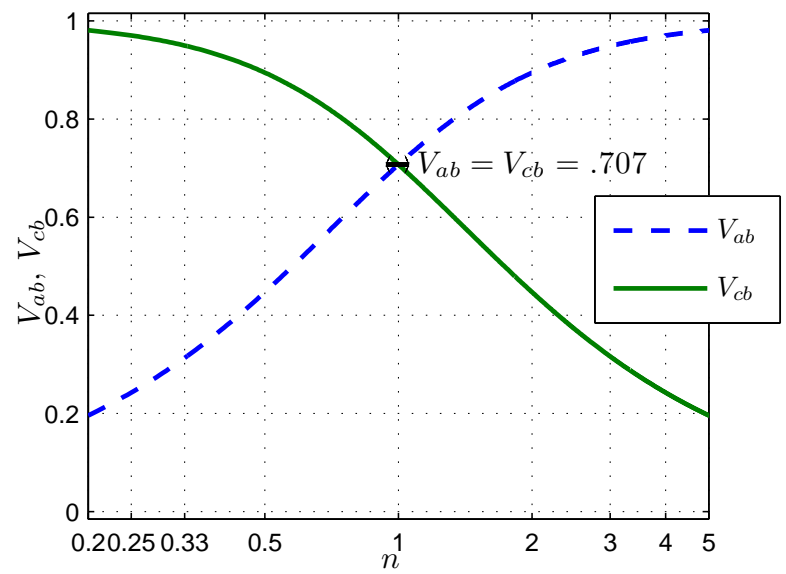

Figura 11: Amplitudes máximas das tensões de linha do conversor com operação na região linear e tensões assimétricas.

A Figura 11 mostra que para operação com tensões assimétricas, a amplitude da tensão de uma das fases da máquina pode ultrapassar o valor de 0,707 pu do barramento CC. Para este caso a tensão $V_{0}^{*}$ deve estar entre os limites impostos de acordo com a equação (9). Na Figura 11 é possível verificar que quando a relação entre as tensões de saída do conversor é igual a $1, n=1$, é obtida a condição de carga simétrica, neste caso é confirmado que a amplitude máxima das tensões de saída moduladas pelo conversor é igual a $0,707 \mathrm{pu}$ do valor do barramento CC.

Para ilustrar a operação com tensões assimétricas, considerase um caso onde a relação $n$ seja igual a 0,64. A Figura 12 ilustra os limites definidos na equação (9) e a tensão $V_{0}^{*}$. Para este caso, a tensão máxima que pode ser modulada é 0,54 para $V_{a b}$ e 0,84 para $V_{c b}$. Na Figura 12 é verificado que $V_{0}^{*}$ atinge o valor limite entre seu valor máximo e mínimo conforme (9).

A Figura 13 apresenta as tensões moduladas nos braços do inversor na obtenção de tensões de linha desequilibradas, enquanto a Figura 14 mostra as tensões de linha moduladas pelo conversor, nesta ilustração, a trajetória das tensões de linha deverá descrever uma elipse conforme mostra a Figura 10. Enquanto que para obtenção de tensões simétricas a trajetória destas é circular, como é mostrado na Figura 3. 


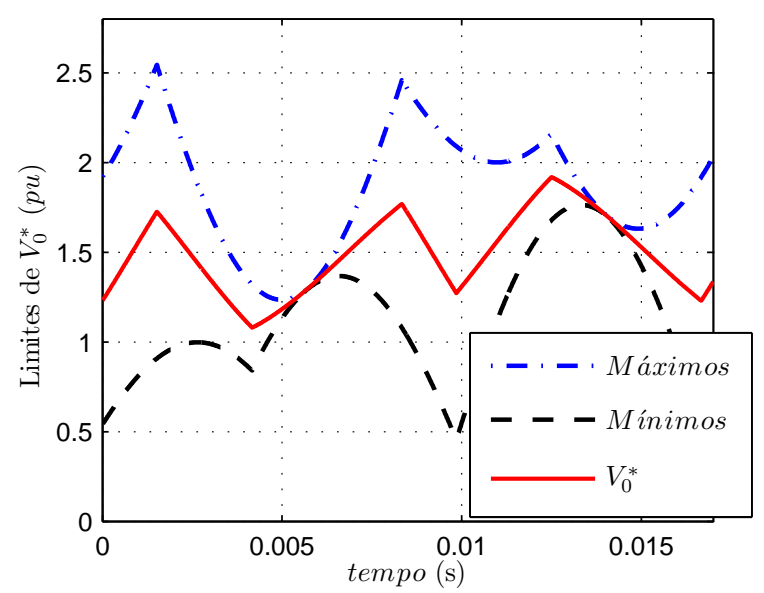

Figura 12: Limites máximos e mínimos de $V_{0}^{*}$ para condição de tensões desequilibradas onde $n=0.64$.

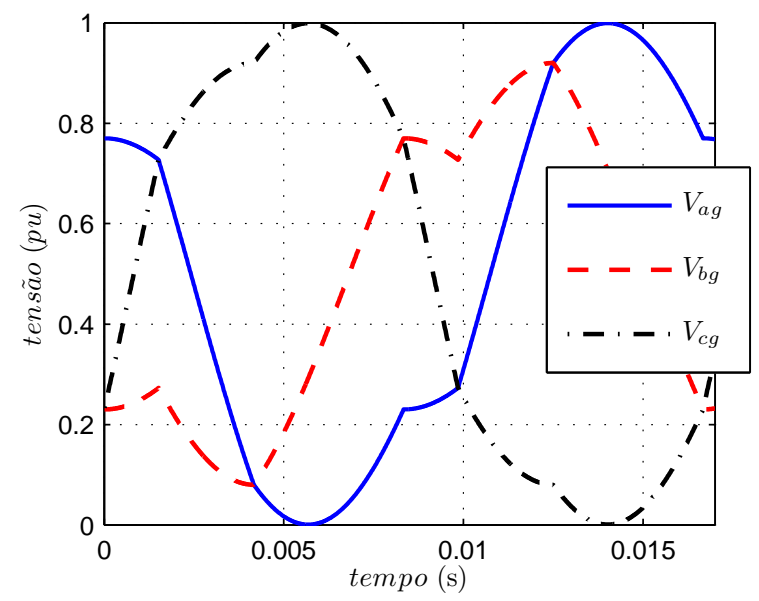

Figura 13: $V_{a g}, V_{b g}$ e $V_{c g}$ para o caso assimétrico.

\section{RESULTADOS EXPERIMENTAIS}

Na obtenção dos resultados experimentais da técnica de modulação proposta foi desenvolvido um ambiente constituído de um inversor trifásico de três braços (vide Figura 1), o qual é comandado por um processador de ponto fixo, DSP TMS320F2812. A freqüência de comutação adotada foi de $5 \mathrm{kHz}$. Na obtenção dos resultados experimentais um motor bifásico assimétrico foi acionado. Os parâmetros elétricos dessa máquina são apresentados na Tabela 2.

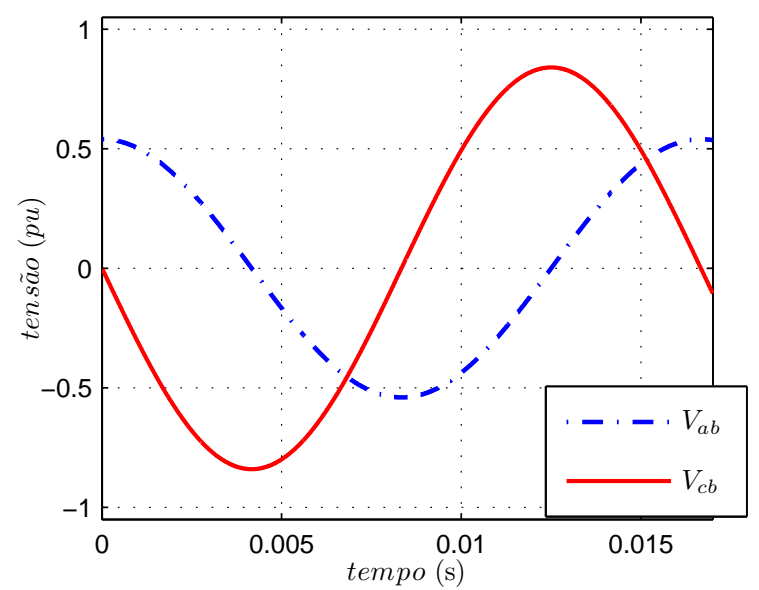

Figura 14: Tensões de linha desequilibradas moduladas pelo conversor.

Tabela 2: Parâmetros elétricos da máquina de indução.

\begin{tabular}{ccccc}
\hline \hline & $R_{s}$ & $R_{r}$ & $L_{s}=L_{r}$ & $L_{m}$ \\
\hline \hline eixo $q$ & $7,0 \Omega$ & $12,26 \Omega$ & $0,2459 \mathrm{H}$ & $0,2145 \mathrm{H}$ \\
\hline eixo $d$ & $20,63 \Omega$ & $28,01 \Omega$ & $0,4264 \mathrm{H}$ & $0,3370 \mathrm{H}$ \\
\hline \hline
\end{tabular}

Os dados de placa da máquina são: $370 \mathrm{~W}, 220 \mathrm{~V}, 1610 \mathrm{RPM}$, 3,4 A.

No primeiro experimento tensões equilibradas com o máximo índice de modulação são aplicadas nos enrolamentos principal e auxiliar do TPIM. Nesse experimento o barramento CC foi limitado em $100 \mathrm{~V}$, logo as amplitudes das tensões médias $V_{a b}$ e $V_{c b}$ devem ser de $70,7 \mathrm{~V}$ em ambos os enrolamentos. A Figura 15 mostra as tensões modulantes $V_{a g}^{*}, V_{b g}^{*}$ e $V_{c g}^{*}$ calculadas pelo método de modulação proposto, enquanto a Figura 16 mostra a tensão de linha $V_{c b}$ medida neste experimento.

A Figura 17 mostra as correntes estatóricas medidas. A corrente no enrolamento auxiliar tem amplitude menor em relação a corrente no enrolamento principal devido a diferença de impedância entre eles. Além disso, a corrente no enrolamento auxiliar é distorcida devido a assimetria da máquina.

No segundo experimento tensões desequilibradas são aplicadas na máquina. A tensão aplicada no enrolamento auxi- 


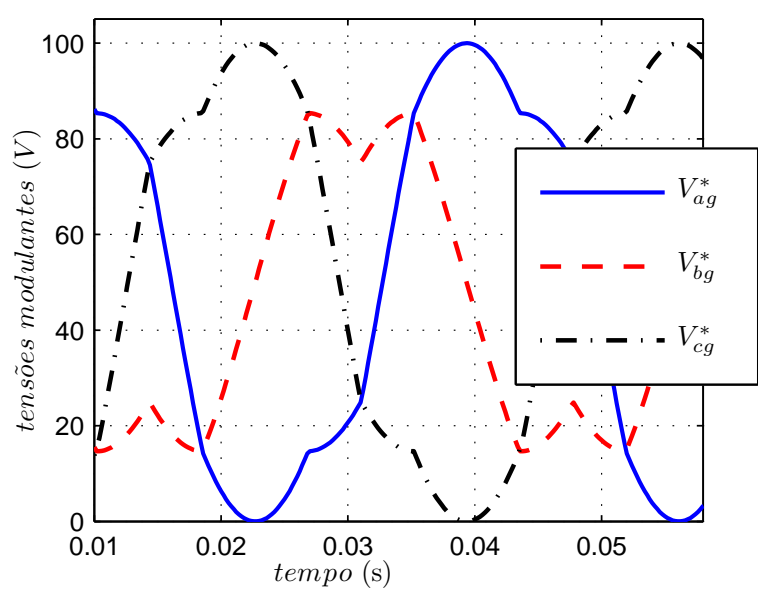

Figura 15: Tensões Modulantes $V_{a g}, V_{b g}$ e $V_{c g}$ para o caso simétrico.

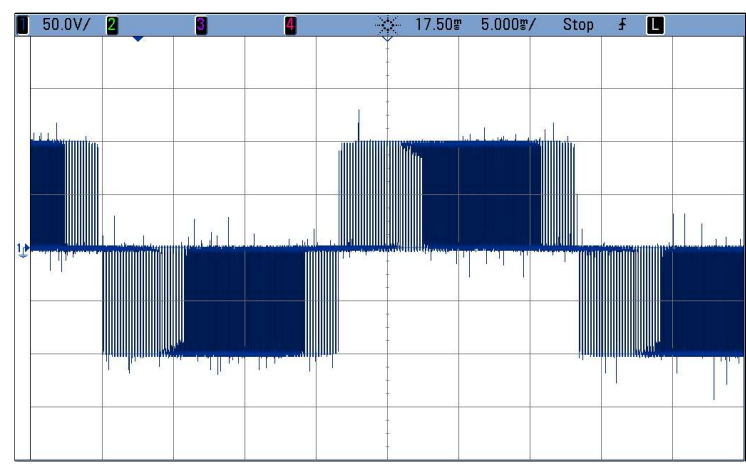

Figura 16: Tensão experimental $V_{c b}$ para o caso simétrico.

liar tem amplitude maior que a tensão aplicada no enrolamento principal para compensar a ausência do capacitor permanente. Então, com a tensão do barramento CC limitada em $100 \mathrm{~V}$, para $n=0,64$ a amplitude da tensão média do enrolamento auxiliar é de 84,01 V enquanto que no enrolamento principal a amplitude da tensão média é de $54,25 \mathrm{~V}$, com defasagem de $90^{\circ}$ entre elas. A Figura 18 mostra as tensões modulantes, enquanto as correntes estatóricas medidas são mostradas na Figura 19. Nesse experimento as correntes medidas apresentam forma quase senoidal, não apresentando distorção significativa na corrente do enrolamento auxiliar, isto implica em redução nas pulsações de torque como descrito em (Charumit and Kinnares, 2009). Neste caso a assimetria característica da máquina é compensada com um aumento da corrente drenada pelo enrolamento auxiliar, consequentemente há uniformalidade nos fluxos estatóricos $q$ e $d$.

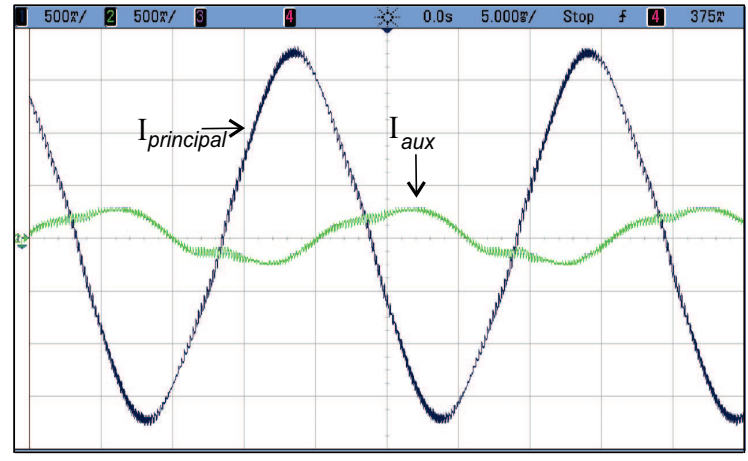

Figura 17: Correntes estatóricas medidas com o TPIM alimentado com tensões equilibradas.

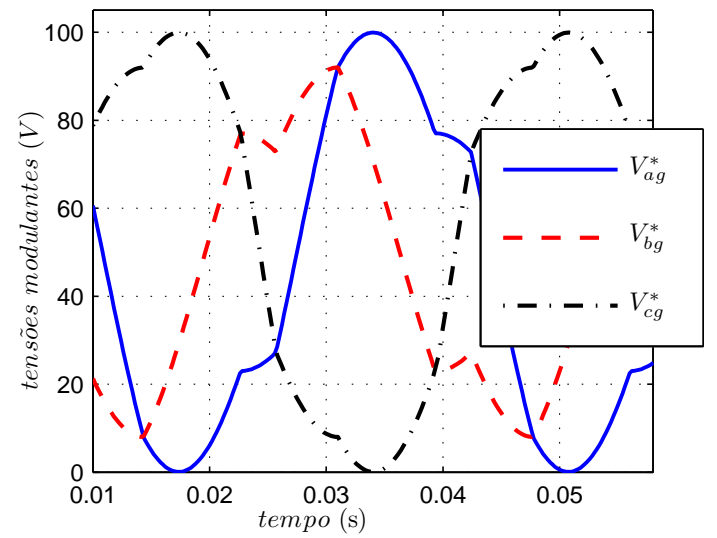

Figura 18: Tensões modulantes $V_{a g}, V_{b g}$ e $V_{c g}$ para o caso assimétrico.

Para verificar o desempenho do método de modulação proposto em um sistema acionando um TPIM com velocidade variável, uma estratégia $V / f$ foi implementada. A Figura 20 mostra a operação em velocidade variável. A Figura 20 (a) apresenta a resposta de velocidade para esse experimento, enquanto a Figura 20 (b) ilustra as correntes estatóricas medidas. A freqüência das tensões estatóricas varia de zero até $60 \mathrm{~Hz}$, enquanto que as amplitudes das tensões médias nos enrolamentos principal e auxiliar variam de zero até $81,37 \mathrm{~V}$ e 126,01 V respectivamente.

\section{CONCLUSÕES}

Neste trabalho foi apresentada uma técnica de modulação PWM aplicada a conversores trifásicos no acionamento de máquinas bifásicas simétricas e assimétricas. $\mathrm{O}$ equacionamento matemático da técnica proposta mostrou que com o uso desta metodologia é possível aplicar tensões com ampli- 


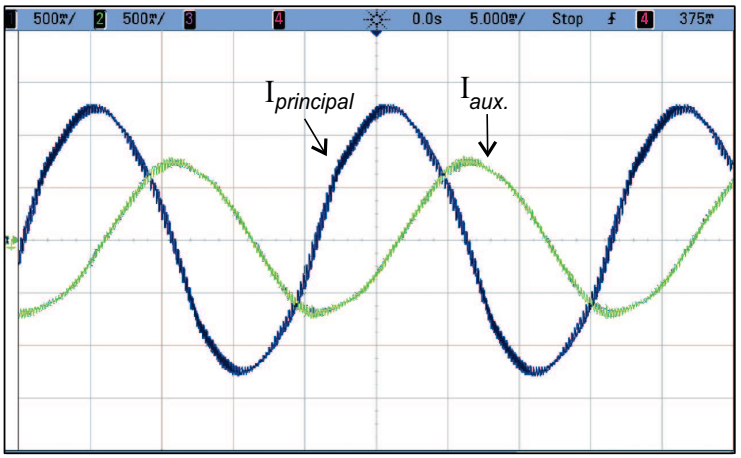

Figura 19: Correntes estatóricas medidas com o TPIM alimentado com tensões desequilibradas.

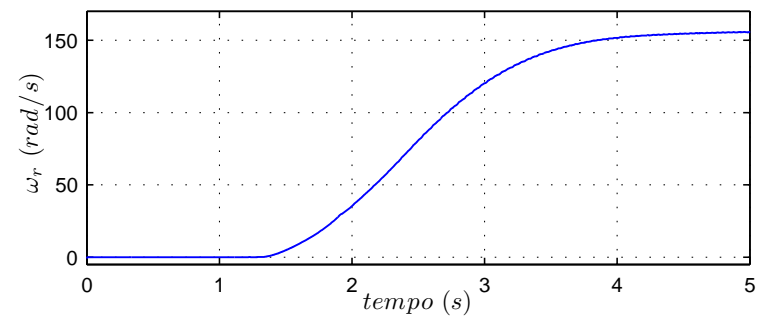

(a)

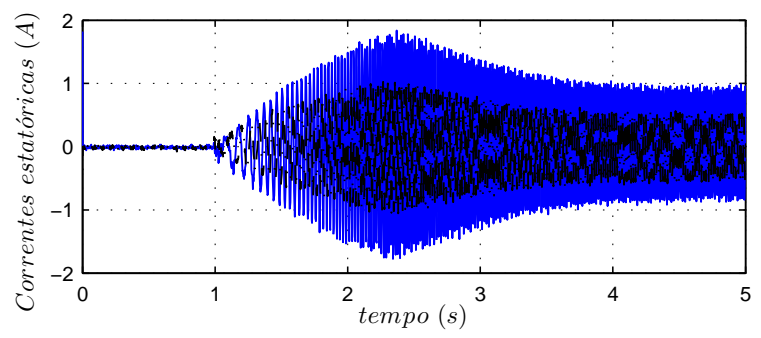

(b)

Figura 20: Operação com velocidade variável para um motor de indução bifásico.

tudes máximas de 0,707 pu do barramento CC nos dois enrolamentos de uma máquina simétrica. Ainda, neste trabalho foi apresentada a operação de conversor no acionamento de cargas assimétricas com tensões desequilibradas. A principal característica da técnica de modulação apresentada neste trabalho é o baixo esforço computacional na implementação da mesma e consequentemente pode ser implementada em controladores digitais de baixo custo. O uso desta técnica ainda pode ser estendido a outros tipos de cargas bifásicas acionadas por conversores de três braços.

\section{AGRADECIMENTOS}

Os autores gostariam de agradecer a CAPES e ao CNPq pelo suporte financeiro.

\section{REFERÊNCIAS}

Amin, A., El Korfally, M., Sayed, A. and Hegazy, O. (2009). Efficiency optimization of two-asymmetrical-winding induction motor based on swarm intelligence, IEEE Transactions on Energy Conversion 24(1): 12 -20.

Blaabjerg, F., Lungeanu, F., Skaug, K. and Tonnes, M. (2004). Two-phase induction motor drives, Industry Applications Magazine, IEEE 10(4): 24-32.

Charumit, C. and Kinnares, V. (2009). Carrier-based unbalanced phase voltage space vector PWM strategy for asymmetrical parameter type two-phase induction motor drives, Electric Power Systems Research 79(7): 1127 - 1135 .

Chomat, M. and Lipo, T. (2003). Adjustable-speed singlephase IM drive with reduced number of switches, IEEE Transactions on Industry Applications 39(3): 819-825.

Corrêa, M., Jacobina, C., Lima, A. and da Silva, E. (2000). Rotor-flux-oriented control of a single-phase induction motor drive, IEEE Transactions on Industrial Electronics 47(4): 832-841.

de F. Campos, R., de Oliveira, J., de S. Marques, L., Nied, A. and Seleme, S. (2007). SVPWM-DTC strategy for single-phase induction motor control, IEEE International Electric Machines Drives Conference, 2007. IEMDC '07., Vol. 2, pp. 1220 -1225.

Donlon, J., Achhammer, J., Iwamoto, H. and Iwasaki, M. (2002). Power modules for appliance motor control, Industry Applications Magazine, IEEE 8(4): 26-34.

dos Santos, E., Jacobina, C., Beltrao de Rossiter Correa, M. and Oliveira, A. (2010). Generalized topologies of multiple single-phase motor drives, IEEE Transactions on Energy Conversion 25(1): 90 -99.

Enjeti, P., Ziogas, P. and Lindsay, J. (1990). Programmed pwm techniques to eliminate harmonics: a critical evaluation, IEEE Transactions on Industry Applications 26(2): $302-316$.

Guerreiro, M., Foito, D. and Cordeiro, A. (2010). A speed controller for a two-winding induction motor based on diametrical inversion, IEEE Transactions on Industrial Electronics 57(1): $449-456$.

Holmes, D. G. and Lipo, T. A. (2003). Pulse Width Modulation for Power Converters: Principles and Practice, Wiley Interscience, IEEE Press.

Holmes, D. and Kotsopoulos, A. (1993). Variable speed control of single and two phase induction motors using a three phase voltage source inverter, IEEE Conference 
Record of the Industry Applications Society Annual Meeting pp. 613-620 vol.1.

Jabbar, M., Khambadkone, A. and Yanfeng, Z. (2004). Space-vector modulation in a two-phase induction motor drive for constant-power operation, IEEE Transactions on Industrial Electronics 51(5): 1081-1088.

Jang, D.-H. (2007). PWM methods for two-phase inverters, Industry Applications Magazine, IEEE 13(2): 50-61.

Jang, D.-H. and Yoon, D.-Y. (2003). Space-vector PWM technique for two-phase inverter-fed two-phase induction motors, IEEE Transactions on Industry Applications 39(2): 542-549.

Jemli, M., Ben Azza, H., Boussak, M. and Gossa, M. (2009). Sensorless indirect stator field orientation speed control for single-phase induction motor drive, IEEE Transactions on Power Electronics 24(6): 1618-1627.

Kinnares, V. and Charumit, C. (2009). Modulating functions of space vector PWM for three-leg VSI-fed unbalanced two-phase induction motors, IEEE Transactions on Power Electronics 24(4): 1135 -1139.

Martins, D. C., Tomaselli, L. C., Lazzarin, T. B. and Barbi, I. (2006). Acionamento do motor de indução bifásico simétrico empregando modulação vetorial, Sba Controle \& Automação 17(3): 356-363.

Pinheiro, H., Botterón, F., Rech, C., Schuch, L., Camargo, R. F., Hey, H. L., Gründling, H. A. and Pinheiro, J. R. (2005). Modulação space vector para inversores alimentados em tensão: uma abordagem unificada, Sba: Controle e Automação Sociedade Brasileira de Automatica 16: 13 - 24 .

Ryan, M., Lorenz, R. and De Doncker, R. (1999). Modeling of multileg sine-wave inverters: a geometric approach, IEEE Transactions on Industrial Electronics 46(6): 1183 -1191.

Vaez-Zadeh, S. and Reicy, S. (2005). Sensorless vector control of single-phase induction motor drives, Proceedings of the Eighth International Conference on Electrical Machines and Systems, ICEMS 2005 3: 18381842 Vol. 3.

Vieira, R. P., Azzolin, R. Z. and Gründling, H. A. (2009). A sensorless single-phase induction motor drive with a MRAC controller, IEEE 35st Annual Conference of Industrial Electronics Society, IECON 2009.

Vieira, R. P., Gastaldini, C. C., Azzolin, R. Z., Pinheiro, H. and Gründling, H. A. (2010). Uma abordagem geométrica para modulação de conversores três braços acionando máquinas de duas fases simétricas e assimétricas, XVIII Congresso Brasileiro de Automática - CBA2010.
Wells, J., Nee, B., Amrhein, M., Krein, P. and Chapman, P. (2004). Low-cost single-phase powered induction machine drive for residential applications, Applied Power Electronics Conference and Exposition, 2004. APEC '04. Nineteenth Annual IEEE 3: 1579-1583 Vol.3.

Zahedi, B. and Vaez-Zadeh, S. (2009). Efficiency optimization control of single-phase induction motor drives, IEEE Transactions on Power Electronics 24(4): 1062 -1070 . 